\title{
Crafting Code: Gender, Coding and Spatial Hybridity in the Events of PyLadies Dublin
}

to appear in S. Luckman and N. Thomas (eds) Craft Economies: Cultural Economies of the Handmade, Bloomsbury.

Sophia Maalsen and Sung-YuehPerng Macquarie University, Australia and Maynooth University, Ireland sophia.maalsen@mq.edu.au and sung-yueh.perng@nuim.ie

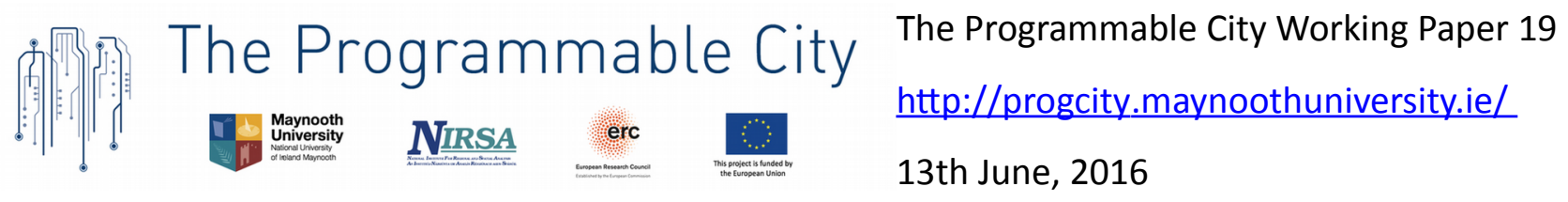

\section{Introduction}

Female friendly coding groups are part of the rapidly expanding hacker and maker movements that experiment with alternative forms of production and innovation (Meyer 2013; Rosner 2014). Specifically in terms of software hacking, it has a long history of tinkering with technology for the pursuit of openness, freedom, transparency and the democratisation of science and technology (Coleman 2013; Lindtner 2015; Maalsen and Perng 2016). While other hackerspaces and makerspaces have become popular in the last decade, spaces that are dedicated to women working on their software or hardware projects are relatively new. In these spaces, organisers and participants make room for the diversity of ideas, genders, goals and practices, and in the process they seek to redefine themselves and their relationships with technology (Fox et al. 2015). 
In this chapter, we follow their work to provide an initial analysis of the gendering of subjectivities and the hybrid spatialities emerging from the work of organising and participating in the monthly 'meetups' of PyLadies Dublin. PyLadies is a global movement that encourages women to code in the programming language of Python, regardless of their levels of skills, purposes of use and professional backgrounds (http://www.pyladies.com/). PyLadies has local chapters across the continents, in cities such as Bangalore, India, Seoul, South Korea and Rio de Janeiro, Brazil, but most of the local chapters concentrate in North America and northern and western European countries and cities. Our research led us to join the Dublin chapter whose inaugural meetup was held in November, 2013, going along to the meetups, as well as learning to code and act in such an environment. In particular, in this chapter we focus on the development of coding subjectivities and hybrid spatiality in the crafting work of PyLadies, and that of the closely related group of Coding Grace, which provides introductory tutorials for participants to learn and incorporate new programming skills into their own work.

There is an increasing discourse around code as craft and we contribute to this discussion. As craft, 'code is about creativity...about engaging with coding as a form of expression and knowledge' (Salmond 2012). For Nafus (2012), engaging in the development of free and open source software is 'craft-like'. Writing software code scratches the programmers' itch of making, sharing, examining and improving the code by removing bugs in it, which motivate the programmers to become involved in such 'communities' and stay. Defining craft, 'an unwieldy beast of a phenomenon' to use Wagner's (2008: 1; Jakob 2012: 3) phrase, is challenging, but as Wagner notes, it is more than just making, but encompasses the political (2012: 3).While technology is often posited as the antithesis of craft and the handmade, repositioning it as a tool for expression and political activism highlights its affinities with craft more broadly. This is perhaps best characterised by hackers and the process of hacking, which Coleman (2013: 98) argues, is a practice in which 'craft and craftiness converge'.

But while we situate coding as a craft we do so to additionally situate its role in knitting together and supporting a community of women that challenge the masculinity of computer programming - thus it is both about making and the political (see also Rosner and 
Fox, 2016). The ethnographic component of the chapter illustrates how during the process of coding and regular meetups, participants develop a strong sense of community through a shared practice of coding. We focus on the practices and processes of making coding subjectivities and spatialites, and by thinking through them as craft work, our approach is similar to Costin's (1998: 3) observations on the ability of craft and crafting to create, maintain, and communicate social identity and relationships through crafting practice and the craft objects produced. We pay particular attention to the technical arrangements, social relationships and material and informational spaces inhabited by Pyladies Dublin, which offers an interesting and fruitful case study as it intersects gender, relations of making and places of making, nested firmly within the social, physical, entrepreneurial and digital worlds.

\section{Coding in gendered spaces}

Crafting an inclusive coding/programming community is valuable work. Research has demonstrated what Corneliussen (2004) refers to as the hegemonic discourse of computing which creates different expectations of relations to computers based on gender (although see Lagesen 2008 for a discussion on different gendered relationships to computer science in Malaysia). Such discourse works to create subject positions in which men are expected to be more knowledgeable, interested and have greater expertise in computer science and to be interested in the technology itself, whereas women are not expected to share this fascination or experience (Corneliussen 2004: 175). This is despite the feminization of computer programming in the early days of computer science (Herbst 2011: 25). Additionally, 'men are associated with computer games, programming and technical tasks, while women are associated with communication, information and writing - tasks that can be described without references to technology' (Corneliussen 2004: 175). Indeed, Corneliussen claims that 'the connection between men and computer skills is so close, that being a man can function as a sign of computer competence' (2004: 177). Computer competence framed as masculine clearly has implications for power, for to 'question the masculinity of computers is questioning our image of masculinity itself: computers are power' (Coyle: 43 cited in Misa 2011: 12). 
In this section, we look at the ways in which inclusive coding communities can help women challenge hegemonic discourses of computing and craft a programmer subjectivity with the help of groups such as PyLadies. Support communities for women within computing have been increasingly seen as a way to retain and recruit women within computing cultures (Gabbert and Meeker 2002), and thus redress the imbalance associated with gendered subjectivities. Toupin (2014) notes the increase in hackerspaces informed by a culture of openness but observes that they remain male dominated spaces, with groups such as women and queers underrepresented. Events which try to redress diversity within these spaces, such as women only nights, are often considered controversial in their exclusiveness which stands in direct opposition to an otherwise open source commitment to an espoused culture of openness (Toupin 2014). However reflecting the hegemonic discourse of computing on a broader computing culture level, the 'openness' of hackerspaces is assumed rather than actual.

Despite this, the hegemony of computing discourse and hackerspaces can however be resisted and reworked. Toupin (2014) demonstrates various endeavours of creating safer and more inclusive spaces for those who do not fit and associate themselves comfortably with dominant hackerspace or computing cultures. Central in creating such spaces is to recognise that the subjectivities of these participants are not fixed, as Corneliussen (2004) details complex strategies that men and women acquired to position their own subjectivities by negotiating with the assumed competences when legitimising their presence in rooms that are of specific gender compositions (e.g. 'a more open room for women' or 'women in a room for men'). We can see similar reworking and crafting of relationships to computers, and programming and coding more specifically in the work of PyLadies, and another female friendly computing space, Coding Grace. We can also see through these efforts to craft a computing community that is inclusive and which offers not only women, but men who do not fit the typical computer expert male subject position completely or comfortably, an opportunity to acquire computing confidence. In an interview Vicky, the organiser of both coding initiatives, said:

Diversity for me initially is more trying to get women into Python as well as any technology but I want to widen the doors too. It doesn't matter what background you are from, culturally, gender wise, religion wise, just anyone who wants to learn 
should have an opportunity to learn. And they should not be afraid to go to technical events.

Technical events here encompass a variety of occasions, from workshops on specific programming techniques to the networking events for programmers. However, these events comprise predominantly white, male participants and can be an intimidating environment for women to attend, regardless of their coding skills. The predominant male presence, the conversation focus of competences and experiences, and the uncomfortable instances and conducts that constitute, or might lead up to, sexual harassment, all discourage females in terms of the participation in technical events and more generally their involvement in computing and other science and technology industries.

Yet crafting safe and inclusive environments is a complex process and can sometimes be hindered by agendas actually intended to encourage openness: the focus on a particular identified minority group may in turn exclude other groups by virtue of this specialised remit. For Vicky, for example, diversity and openness involve issues much broader than gender. During the interview she re-enacted a conversation she had with a company which potentially can provide sponsorships for events that she plans to promote as 'diversity friendly':

... 'diversity, does that mean you are open to everyone?' I said, 'yes.' He said, 'but our remit is for women in tech.' I said, 'ok I will just keep it female friendly' So we are not quite ready to call it diversity friendly, so when you explain diversity for me it is open doors to anyone who wants to come and learn so it is not gender only.

Vicky's aim to create open and diverse coding spaces however is paradoxically restrained by initiatives to enhance gender equality within computing cultures. Diversity, being open to everyone, is perceived differently in the excerpt above and significantly reduced in scope to the focus on 'women'. The broader diversity label challenges people's perceptions concerning for whom computing cultures are promoted and shaped, and is particularly the case when Vicky was sourcing event sponsorships from the private sector. At the same time, aligning with female friendly remits, is itself met with resistance from predominantly male programmers who see such events as divisive, as reflected in both Vicky's experience and 
Toupin's (2014) aforementioned work. Groups such as PyLadies and Coding Grace seek to build inclusive computing cultures; however their work encounters resistance of the broader computing community which view female friendly events as exclusive or divisive and hindered by perceptions and remits that narrow down the scope of diversity.

Crafting an inclusive community is also an ongoing and reflexive process. This process is dependent on the individuals within the community and the work they do to perform their subjectivity within computing cultures. As participants in PyLadies and Coding Grace learn to code, they rework and negotiate their own subjectivity in the process. Resisting the hegemonic discourse of computing and gender ascribed subject positions is however, a continuous practice. Participants can learn to code and become a competent programmer but still feel unable to inhabit a subject position of computing competence. This is most evident in a conversation between Vicky and another participant. The participant has removed the pre-installed Windows to her laptop, which now runs on Linux and still encounters problems. As Vicky described,

She fixed her own machine -- she is working on Linux - and she is wondering what is going on. Basically she has been tinkering with her laptop, she has been coding, she has been going to tutorials, she is a coder but she just doesn't admit it. But she is compiling stuff away, doing this and that and she says she is not a programmer.

Inhabiting the position of 'programmer' or believing one to be a competent coder, is complex identity work. It becomes a subjective matter, or a matter of subjectivity. As Vicky continues to discuss, there is a multiplicity to coder subjectivity which influences how individuals see themselves as fully or partially being that subject. Some are more closely related to coding, including confidence, competence and experience, and others depend upon how individuals recognise the relationships between code, work and identity. The same participant does not see herself as a coder partly due to that her day work does not involve much coding, and similar situation applies to those who write codes only as part of their work: 
She was saying in the work she does at the time she was contracting, there wasn't much coding involved and I think a lot of people have this perception that they are not coders. Researchers or data people say, I am not a coder.

But for Vicky, these are all valuable experiences of writing code and qualify these individuals as coders:

You are a coder, you are writing Python code, you are analysing data, you are using libraries. You are writing code so you are a programmer! You could be other stuff as well, you are a data analyst, but you are a programmer because you code stuff. I think it is people's perception and herself, I tried to convince her that you are a coder!

These conversations show that maker identities (including programming/coding) are fluid and influenced by a subject's 'ability to use and extend tools, adopt an adhocist attitude to projects and materials, and to engage with the broader maker community' (Toombs, Bardzell and Bardzel 2014). The participant discussed above clearly demonstrates the traits listed by Toombs et al. but still has difficulty in accepting her subject position as oppositional to that prescribed by the hegemonic discourse of computing. Despite being able to write code, the participant still has difficulties in authoring her identity as a programmer. Subject positions are therefore influential on an individual's computing competence, however it also illustrates that these identities can be negotiated. This negotiation is a complex process and at times there are discrepancies between an individual's ability and their self-perception, but inclusive spaces such as PyLadies and Coding Grace can redress this imbalance and help the individual grow into their 'programmer' subjectivity.

\section{Crafting hybrid coding spaces}

In the case of PyLadies Dublin, organising inclusive, friendly and supportive spaces for coding forms another aspect of their valuable work. The 'matters' produced through these spaces are the amalgamates of programmers, gatherings, spaces and cultures that support 
one another in their engagements with a particular programming language and with the tech culture and industry more generally. These coding groups and spaces are in some ways comparable to a 'mobile sewing circle' where participants meet in person and embroider mobile phone text messages to make 'matters': the patchwork and the sharing and articulating of personal stories, experiences and concerns emerging as a result of engaging with each other's life, messages and sewing tools (Lindström and Ståhl 2012). The practices of documenting the code they work on online, organising gatherings that foreground mutual support, and making themselves available to each other, offering skills and providing encouragement, enhance wider, supportive and more collaborative engagement with technology. To demonstrate how such spaces and communities are developed, we build on an emerging field of studies that examine a variety of hackerspaces, makerspaces and DIY Labs and their social, cultural, technological and economic significance (e.g. Meyer 2013; Lindtner et al. 2014). In developing the analysis, we observe a wide range of informational, emotional, material and embodied practices that make PyLadies communities and coding spaces for developing alternative coding culture.

PyLadies' meetup venues differ each month, and Meetup.com and Facebook pages are set up for event announcement and coordination. Like them, we look up event details from these pages before travelling together on train to find the venues. On a bright evening in June 2014, this research journey led us to the creative and historic quarter of Dublin. Arriving in Dublin and with a printed map in hand, we embarked on the walk that led us to the streetscape dominated by elegant four-story Georgian buildings in an area developed in the 18th century for merchant houses but now transformed into a bustling commercial quarter. The venue was in an incubator space in that quarter, converted from one of the terraced Georgian townhouses in a busy street full of niche retailers, and that was where we spent the next two hours, writing codes together with our fellow programmers.

Like this meetup, many others were hosted by technology companies that are Dublin-based or have headquarters or branches here. But some of the venues were trickier to find compared with this pleasant short walk. It takes each participant considerable effort and determination to join because the meetup is after work, involves more than listening to talks, and requires actual code writing. Furthermore, these venues can be outside of the central area and difficult to reach by public transportation, adding onto the difficulty of 
travel during peak hours. Accordingly, participants are always in high spirit to see others joining them, and the sense of a 'coding community' grows as the members turning up to meetups recurrently while they move across Dublin each time they meet.

In addition to this conviviality, the material arrangements of the room and embodied interactions occurring there have been important to develop a distinctive, supportive atmosphere even though coding can be an individual activity. At a practical level, coding can be a very personal, considering that a programmer has to organise her own understanding and reasoning about life world situations, and translate them into software codes according to specific rules set by the programming languages being used. However, regardless of the different locations or layouts of the venue, PyLadies meetups often comprised of two or three tables joined together so that people could sit around them to form a 'group'. The group felt social and lively when participants were arriving. Sounds of greeting and catching up with each other infused a social element to the gathering. By contrast, when participants focused on their codes, the time in the room proceeded in a way that felt as if it ceased to exist, that it was not even noticeable. For these participants, it was because they were so engrossed into their thoughts, codes, fingers and screens, finding out how to do the syntax right or looking up additional information online. PyLadies has a shared project of building their own website and connect it with the existing Meetup.com or Facebook pages. But contributing to the collective project is not a prerequisite to participate in the meetup. Instead, it is mostly intended for those who want to come along but do not have a specific project in mind. Indeed, the organiser has encouraged participants, repeatedly, to bring their own projects to the meetup, as long as they get to spend time on coding 'in a likeminded environment instead of ... binge-watching tv episodes which seems to be the norm as folks want to chill and relax even though they plan to learn something new like coding' (Vicky, Personal communication). By seating the participants together but having both individual and shared projects at the meetups, these arrangements make flexible space to encourage participants to learn coding in their own ways and to give or receive help to troubleshoot problems during their engagement with new codes, as the following interlude occurring at the same meetup demonstrates.

To discuss technical problems in a shared space often means interruption to other participants, and it requires considerable embodied boundary work to create spaces for 
discussion and collaboration. As the meetup became quiet when participants started working on their projects, one participant broke the silence and stillness, very gently, and tried to ask the mentor a question. Immediately, she felt abashed for having to raise her voice for sending it across the whole room during the initial, short exchange of words. Becoming really conscious about her 'intrusion' into their own, shared space, she moved herself to sit next to the mentor on the far side of the room, to continue their conversation. Even though other participants did hear the conversation, they did not seem to mind the discussion and carried on with their own projects. This short 'disruption' demonstrates the in situ practices of producing collaborative working spaces critical for sustaining the coding community. These spaces are not designated or fixed; instead they emerge and disappear when troubles occur and become fixed. They can be short, but can also linger when technical issues become followed by unanticipated but engaged discussion on issues not restricted to technical ones. For example, the issue of 'coding subjectivity' as we discussed above is a recurrent theme, and is one through which participants explore, as a group, their assumptions about the skills and identities associated with an appropriate 'programmer'. Accordingly, these highly negotiable and permeable boundaries between technical and social issues and between individual and shared issues matter for PyLadies' coding spaces because they foster skill and knowledge sharing and reflections on personal and social conditions in relation to male-dominant coding subjectivity.

Apart from such in situ practices, PyLadies participants also use an online collaborative note-taking tool to share their own project ideas, progresses and suggestions. These documents of project ideas and progresses act as more than archives, and become a dynamic place of project development facilitated by the support network of the Pyladies community. In the beginning, PyLadies tried to assemble necessary Python frameworks to build their own website, as well as for the beginners to learn the language. The online notes have detailed how to create and activate a virtual environment, set up Python modules, frameworks or libraries, and connect to Meetup.com's API to make the functions available on PyLadies' Meetup pages (e.g. RSVP an event) equally available in their website. These notes allow the participants to re-create the process outside of the meetup, or to catch up if they fall behind or could not participate in that particular session. In more recent meetups where participants work more on their individual projects, the provision of technical details can still be found. For instance, one of the participants has an idea of accessing and 
obtaining the data about available bikes from the bike-share scheme of Dublin Bikes, and visualises and publishes the availability for its users. This attracts the interest of another participant who then goes on to find out where and how to access the data and how to parse the data obtained.

Taking into account the online, material, informational and embodied arrangements around PyLadies' spaces of coding, we argue that PyLadies itself is craft work that weaves together supportive software code writing practices and more inclusive spaces to 'widen the door' for those who only have restricted accesses and resources to programming. Finding venues and sponsorships to organise monthly gatherings can be repetitive, but still require careful and creative consideration to enhance alternative sociotechnical cultures and imaginaries around programming and to promote more supportive, collaborative, encouraging and transformative cultures. The spaces of coding emerge through participants engaging in individual projects, sharing knowledge and experiences among themselves, and motivating each other. In turn, they reconfigure coding cultures by crafting technically, socially and emotionally supportive spaces to work. The participants dedicate time to themselves to write codes, develop mutual encouragement, provide company to each other, and lend helping hands only when necessary, highlighting that they are a group of competent, individual, female programmers. But they do more than producing code. The production of code also provides an opportunity to reevaluate and reconfigure their sense of self. Through continual engagement in the group, participants learnt to not only code but to learn to identify themselves as coders/programmers, an identity that many initially felt unworthy to inhabit. In the process they have crafted a cultural shift, strongly aligning with the political aspect of craft. In this way, female coding subjectivities become inseparable from how they perform hybrid sociospatial relationships around coding. The multiple places where coding and gatherings take place are reconfigured and become alternative 'geographies of display' (Wakeford 1999), ones that focus less on scripting gendered bodies and identities and instead demonstrate possibilities of hacking, diversifying and reconfiguring hegemonic coding culture, subjectivity and spatiality. 


\section{Conclusion}

In this chapter we have explored the spatial, performed and gendered dynamics of crafting code and an inclusive coding community. Initially we had intended to address coding/programming as a craft and to attend to the dynamics that support that process. We discovered through our research, that much more than code was being crafted. Through engaging with PyLadies and Coding Grace, participants have made, negotiated and reworked their subjectivities as coders and programmers, in the process not only gaining the skills to code, but also contributing to the development of an inclusive and supportive computing community. This process was, however, continuous and reflexive, with individual subject positions fluid and performed relative to the space. On a broader level these actions played out to build networks and links outside of the two female friendly coding meetups. The organisation of the events required partnerships with companies invested in increasing women's participation in computing cultures, and as such the location of the meetups was changeable, dynamic and fluid. The spatial dynamics of the meetups were an important element in providing a supportive coding environment. Whilst positive steps had been made towards encouraging participation within computing cultures, there remained resistance to increasing diversity more broadly. As such, those involved with the groups see the project as far from complete. However, the case studies discussed here demonstrate that it is through the act of coding and participating in such spaces, that individual subjectivities are reworked and remade, and that inclusive spaces are built, one event, and one line of code at a time.

\section{Acknowledgements}

The research for this paper was conducted under the Programmable City project, funded by a European Research Council Advanced Investigator award (ERC-2012-AdG-323636SOFTCITY). We are grateful for the participants of PyLadies Dublin and Coding Grace for their encouragement and generosity along our research journey. 


\section{References}

Coleman, E. G. (2013), Coding Freedom: The Ethics and Aesthetics of Hacking, Princeton, NJ: Princeton University Press.

Corneliussen, H. (2004), “I don't understand computer programming, because I'm a woman!" Negotiating gendered positions in a Norwegian discourse of computing', in K. Morgan, C.A. Brebbia, J. Sanchez and A. Voiskounsky (eds), Human Perspectives in the Internet Society: Culture, Psychology and Gender, 173-182, Southampton, Boston: WIT Press.

Costin, C. (1998) 'Introduction: Craft and Social Identity', Archaeological Papers of the American Anthropological Association, 8 (1): 3-16.

Coyle, K. (1996), 'How hard can it be?', Wired Women, Seattle: Seal Press.

Crang, M., Crosbie, T. and Graham, S. (2007), 'Technology, time-space, and the remediation of neighbourhood life', Environment and Planning A, 39 (10): 2405-22.

Fox, S., Ulgado, R. and Rosner, D. (2015), 'Hacking culture, not devices: Access and recognition in feminist hackerspaces', in Proceedings of Computer-Supported Cooperative Work, 56-68, Vancouver, BC, Canada,.

Herbst, C. (2009), Sexing Code: Subversion, Theory and Representation, Newcastle: Cambridge Scholars Publishing.

Jakob, D. (2012), 'Crafting your way out of the recession? New craft entrepreneurs and the global economic downturn', Cambridge Journal of Regions, Economy and Society, doi:10.1093/cjres/rss022

Lagesen, V.A. (2008), 'A cyberfeminist utopia? Perceptions of gender and computer science among Malaysian women computer science students and faculty', Science, Technology, and Human Values, 33 (1): 5-27.

Lindström, K and Ståhl Å (2010), 'Making private matters public in temporary assemblies', CoDesign: International Journal of CoCreation in Design and the Arts, 8 (2-3): 14561.

Lindtner, S. (2015), 'Hacking with Chinese characteristics: The promises of the maker movement against China's manufacturing culture', Science, Technology and Human Values, 40 (5): 854-79. 
Lindtner, S., Hertz, G.D. and Dourish, P. (2014), 'Emerging sites of HCI innovation: Hackerspaces, hardware startups and incubators', in Proceedings of CHI, 439-48, Toronto, Canada.

Maalsen, S. and Perng, SY. (2016), 'Encountering the city at hacking events', in R. Kitchin and SY. Perng (eds) Code and the City, London: Routledge.

Meyer, M. (2013), 'Domesticating and democratizing science: A geography of do-it-yourself biology', Journal of Material Culture, 18 (2): 117-34.

Misa, T.J. (2011), Gender Codes: Why women are leaving computing. New Jersey: Wiley \& Sons.

Nafus, D. (2012), “Patches don't have gender": What is not open in opensource software', New Media \& Society, 14 (4): 669-83.

Rosner, D. K. (2014), 'Making citizens, reassembling devices: On gender and the development of contemporary public sites of repair in Northern California', Public Culture, 26 (1): 51-77.

Rosner, D. K. and Fox, S. (2016), 'Legacies of craft and the centrality of failure in a motheroperated hackerspace', New Media \& Society, doi: 10.1177/1461444816629468.

Salmond, M. (2012), 'Code as Craft', Journal of the New Media Caucus, 8 (1), http://median.newmediacaucus.org/spring-2012-v-08-n-01-caa-conferenceedition-2012-code-as-craft/ [6 May 2016]

Toupin, S. (2014), 'Feminist hackerspaces: The synthesis of feminist and hacker cultures', Journal of Peer Production, available from http://peerproduction.net/issues/issue5-shared-machine-shops/peer-reviewed-articles/feminist-hackerspaces-thesynthesis-of-feminist-and-hacker-cultures/ [17 September 2015]

Wagner,A. (2008) 'Craft: It's What You Make of It'. In F. Levine and C. Heimerl (eds.) Handmade nation: The rise of DIY, art, craft, and design. New York: Princeton Architectural Press.

Wakeford, N. (1999), 'Gender and the landscapes of computing in an Internet café', in M. Crang, P. Crang, and J. May (eds.) Virtual Geographies: Bodies, Space and Relations, 178-201. London: Routledge. 